\title{
NOUVELLES PERSPECTIVES POUR L'ÉDITION ET LA LEXICOLOGIE DES POÈMES DE NICANDRE
}

The paper deals with the editorial and lexicographical consequences of a revision of the role to be played in the edition of Nicander's poems by the manuscript Parisinus Bibliothecae Nationalis Supplementum graecum 147. From a renewed examination, it appeared indeed that the text of the Parisian codex results from an erudite revision, made at the end of Antiquity/beginning of the Proto-byzantine epoch. Instead of presenting thus a better text than the consensus of all the other manuscripts, it gives a lecture of Nicander's poems, the elements of which have been further inserted in the text. By means of the presentation of cases, we stress the fact, analysing above all its impact on the edition and the lexicology of Nicander's poems.

\section{Introduction ${ }^{1}$.}

Des Thêriaka et Alexipharmaka du poète grec alexandrin Nicandre dit de Colophon (II ${ }^{\mathrm{ème}}$ siècle avant notre ère) ${ }^{2}$, le premier traite de la toxicologie des envenimements et le second de celle des empoisonnements ${ }^{3}$, selon une mé-

1 Cet article a été rédigé dans le cadre du programme de recherche PB95-0138 appuyé par la DGICYT, à laquelle je tiens à manifester ici ma reconnaissance.

2 Sur Nicandre, voir en dernier lieu et avec la bibliographie antérieure, l'article de Touwaide 1991, signalé dans la bibliographie en fin de l'article. Pour la clarté, nous avons renvoyé dans l'Appendice bibliographique ci-dessous les références des travaux relatifs à Nicandre que nous citons dans les notes du présent travail, au contraire des ouvrages et articles qui ne portent pas de façon spécifique sur ce thème, qui sont cités avec leur référence complète loco opportuno. De la sorte, cette bibliographie nicandréenne constitue un premier outil de travail récent et mis à jour sur la question (même s'il n'est pas exhaustif), en attendant la publication d'une Bibliographie de Nicandre, que nous avons d'ores et déjà mise en chantier.

3 Texte grec des deux poèmes avec traduction anglaise et notes de commentaire dans Gow \& Scholfield 1953; traduction française des Alexipharmaka dans Jacques 1955 (avec brèves notes de commentaire); traductions espagnoles: Vera 1970, pp. 468-507 (qui, d'après 
thode médicale typique ${ }^{4}$. Les deux poèmes se caractérisent par une écriture qui vise à insérer leur matière technique dans la langue, le vers, la musique et la réalité d'Homère, provoquant par là non seulement un phénomène d'autoidentification du lecteur par référence au texte fondateur du discours culturel grec antique, mais aussi son implication dans un sujet cependant aride et impersonnel, par la fusion délibérément organisée du monde des venins et poisons dans celui d'Homère et par l'insertion de la matière toxicologique dans celle de l'épopée 5 .

Largement imités dès l'Antiquité ${ }^{6}$, les deux poèmes furent transmis par une tradition manuscrite abondante 7 , à tout le moins eu égard à l'étrangeté du propos, pour être édités dès 1499, par nul autre qu'Aldo Manuzio (vers. 14491515), en appendice à certains exemplaires de l'édition du texte grec d'un

l'indication de la p. 468, semble établie à partir des éditions de J. G. Schneider 1792 et 1816), et Touwaide 1997/2 (avec présentation et notes de commentaire); traduction allemande (établie sur l'édition de Schneider 1856, avec notes d'identification des animaux, des toxiques, des plantes médicinales et matières médicales) dans Brenning 1904. Pour des analyses techniques des poèmes, avec identification des animaux venimeux et des produits toxiques, ainsi que d'un certain nombre des plantes médicinales, voir Scarborough 1977 et 1979, à compléter par Bodson 1986. Par ailleurs, pour une évaluation pharmacologique sommaire des Thêriaka, voir Skaltsa-Diamantis \& Philianos 1994.

Il convient de noter ici que Jean-Marie Jacques a préparé une nouvelle édition critique dans le cadre d'une thèse de doctorat présentée en France, qu'il ne nous a pas été donné de consulter; par ailleurs, nous-même, nous en préparons une, qui fait suite à nos deux thèses sur Nicandre (Touwaide 1975 \& 1977) et à l'analyse renouvelée que nous avons donnée du manuscrit Parisinus supplementum graecum 247 (Touwaide 1997), dont nous présentons des éléments dans le présent article.

4 Voir Touwaide 1991/2.

5 Voir Touwaide 1991, avec la bibliographie antérieure.

6 Sur cette question, voir, par exemple et en ordre chronologique, les travaux de Barwick 1925, Cazzaniga 1959, 1960, 1960/3, Arrigoni 1970, Geymonat 1970 et Gualandri 1970, avec une première synthèse dans Knoefel \& Covi 1991, passim.

7 Pour des inventaires des manuscrits de Nicandre, voir, dans l'état actuel de la bibliographie O. Schneider 1856, pp. 212-216; Gow \& Scholfield 1953, p. 9-15, qui reproduit fondamentalement le travail de Schneider, sans élément nouveau; et, plus récemment et plus complet, R. E. Sinkewicz, Manuscript Listings for the Authors of Classical and Late Antiquity (= Greek Index Project Series, 3), Toronto, 1990, p. L23, N23 \& B 24., qui inventorie les manuscrits au départ des catalogues imprimés, sans les avoir examinés de façon personnelle et directe. Dans le cadre de notre édition critique en préparation (voir note 3), nous avons établi un nouvel inventaire des manuscrits, qui nous a permis d'identifier un nombre important de codices, dont plusieurs avaient échappé aux recensements précédents. Nous espérons publier cet inventaire de façon séparée, avec une première description des manuscrits. 
ouvrage dont ils pouvaient constituer comme un complément, le Traité de matière médicale de Dioscoride ( ${ }^{\mathrm{er}}$ siècle de notre ère) ${ }^{8}$. Plusieurs fois réédités par la suite ${ }^{9}$, abondamment lus, commentés et traduits à la Renaissance ${ }^{10}$, ils connurent par après une défaveur d'où ils ne sortirent qu'il n'y a guère: la dernière édition en date, due à Gow et Scholfield ${ }^{11}$, attira en effet l'attention sur ce texte rare et curieux et suscita -ou, du moins, contribua à le faire - les travaux des Cazzaniga (et de ses disciples), Jacques et Papathomopoulos ${ }^{12}$, ainsi que les nôtres ${ }^{13}$.

Un récent examen du manuscrit le plus ancien actuellement connu du texte grec des deux poèmes, le Parisinus Bibliothecae Nationalis Supplementum graecum 247 effectué dans le cadre de la publication en fac-similé de ce codex ${ }^{14}$, nous a porté à soumettre à un examen critique l'édition des deux poèmes, et notamment l'usage fait du manuscrit parisien. Il est apparu ainsi que la primauté accordée à cet exemplaire dans l'établissement du texte, héritée de l'édition déjà mentionnée d'O. Schneider, résulte moins d'une réelle supériorité du manuscrit que d'une forte différence par rapport à ceux qui étaient connus jusque là, et, partant, par rapport aux éditions antérieures, dont les dernières en date étaient alors celles de J. G. Schneider ${ }^{15}$. Car, une archéologie philologique du texte du Parisinus en a révélé la nature composite, due, essentiellement, au fait que des gloses et variantes s'y sont substituées à des leçons originales; sur ce matériel, sont ensuite venues se greffer des fautes de majuscules et de minuscules, qui ont défiguré le texte et en ont obéré jusqu'à l'intelligibilité, suscitant dans le même temps des tentatives de restauration; celles-ci ont cependant été rarement couronnées de succès et se sont soldées par des paradiorthoses qui ont encore ajouté à l'hétérogénéité du texte.

Telle est la problématique que nous nous proposons d'aborder ici, concentrant l'attention moins sur l'histoire et la constitution du texte du Parisinus

8 Nicandre 1499. Sur cette édition, voir notamment: L. Hain, Repertorium bibliographicum in quo libri omnes ab arte typographica inventa usque ad annum MD typis expressi ..., 2 vol., Stuttgart \& Paris, 1826-1833 (réimpression: Milano, 1948-1950), num. 6257; Gesamtkatalog der Wiegendrucke, vol. 7, Leipzig, 1938, col. 464-466, num. 8435.

9 Pour les éditions, voir L. Choulant, Handbuch der Bücherkunde für die ältere Medizin ..., Leipzig, 1841 (réimpression: Graz, 1956), p. 62-65

10 Présentation générale, mais non exhaustive dans Knoefel \& Covi 1991, passim.

11 Gow \& Scholfield 1953, avec des notes lexicographiques préparatoires dans Gow 1951.

12 Voir dans l'Appendice bibliographique, sub 4 et 5 (Editions des paraphrases et scolies), 6 (Index et concordances) et 7 (Etudes et travaux).

13 Voir nos thèses de 1975 et 1977, ainsi que les articles de 1991 et l'ouvrage de 1997.

14 Voir Touwaide 1997.

15 Voir J. G. Schneider 1792 et 1816. 
que sur leur incidence sur l'édition des deux poèmes et, par conséquent, sur la lexicologie nicandréenne et, de façon plus générale, sur la lexicologie du grec antique. Pour ce faire, nous procéderons en trois temps: après avoir présenté l'histoire des éditions récentes du texte et de la place prise par le Parisinus dans l'ecdotique du texte de Nicandre, nous mettrons en évidence le changement que la nouvelle évaluation du texte du manuscrit provoquera dans l'édition des poèmes de Nicandre, pour nous arrêter, ensuite, sur les modifications que cette stratégie ecdotique entrainera dans le secteur de la lexicologie grecque ancienne.

$\mathrm{Du}$ point de vue méthodologique, les questions soulevées ici ne pourront pas être traitées de façon exhaustive en raison de l'ampleur du matériel; par conséquent, elles le seront au moyen d'exemples significatifs des faits relevés au cours de notre examen, le reste de la documentation étant destiné à être communiqué dans une publication ultérieure, qui pourrait être sinon la nouvelle édition critique des deux poèmes que nous avons annoncée ci-dessus, du moins une histoire de leur texte, accompagnée, quel que soit l'objet de cette publication, du nouvel index du texte grec des Theriaka et des Alexipharmaka que nous avons préparé ${ }^{16}$.

\section{Les éditions des poèmes de Nicandre et le Parisinus.}

Edités à la fin du XVIII ème et au début du XIX ${ }^{\text {ème }}$ siècle ${ }^{17}$, les deux poèmes le furent une nouvelle fois pas même cinquante ans après ${ }^{18}$. En cause, la découverte du Parisinus. Car le manuscrit, qui, jusqu'à la Révolution, avait appartenu à l'Abbaye parisienne de Saint-Germain-des-Prés, devint alors patrimoine de la nation et fit son entrée dans les collections de la Bibliothèque Nationale ${ }^{19}$. Inconnu ou pas accessible avant, il fut ainsi mis à la disposition du monde savant et vint à la connaissance de U. C. Bussemaker, qui en signala l'existence au monde savant ${ }^{20}$.

Sans doute à la suite de cette découverte, quoique, à vrai dire, il n'en dise rien ni n'en laisse rien transparaître, $O$. Schneider entreprit la rédaction d'une

16 Cet index, resté inédit jusqu'ici, a été constitué dans le cadre de la préparation de nos deux thèses de 1975 et 1977. Etabli sur l'édition de Gow \& Scholfield 1953, il demande, pour être publié, d'intégrer les données résultant de notre nouvelle analyse du Parisinus, soit d'introduire les leçons divergentes des manuscrits ou groupes de manuscrits, essentiellement $\Omega$ (le consensus des manuscrits opposés au Parisinus) et $\Pi$ (le Parisinus).

17 Voir J. G. Schneider 1792 et 1816.

18 O. Schneider 1856.

19 Voir Le patrimoine libéré, Paris, 1989, num. 135.

20 Bussemaker 1849, p. V. 
nouvelle édition critique du texte de Nicandre, qui fut publiée par Teubner. Quoiqu'il n'ai pas consulté personnellement le manuscrit, ne le connaissant qu'à travers une collation effectuée à son intention par H. Keil, il l'évalua de la sorte: après avoir énuméré les vingt-deux autres exemplaires du texte de Nicandre qu'il prétendait avoir consultés, il déclara d'entrée de jeu qu' «aucun des autres codices ne pouvait en égaler la qualité» ${ }^{21}$; plus loin, il explicita cette opinion, affirmant que le Parisinus, auquel fut attribué le sigle $\Pi$ qu'il porte depuis lors dans la bibliographie, «l'emporte sur tous les autres tant par son ancienneté que par sa qualité», d'abord parce qu'il présente des leçons qui concordent avec celles attestées par Galien, (129 - après 216 de notre ère [?]) et Athénée de Naucratis (III ${ }^{\text {ème }}$ siècle de notre ère); ensuite, parce qu'il donne, pour les passages corrompus, soit la leçon correcte, soit une leçon à partir de laquelle il est possible de retrouver le texte original; enfin, parce qu'il offre la plupart du temps des leçons jugées par Schneider plus savantes et plus raffinées que celles des autres manuscrits, d'une façon qui, dit-il en outre, est conforme au style de Nicandre ${ }^{22}$. Ces affirmations, à l'appui desquelles aucun élément concret n'était allégué, portèrent $\mathrm{O}$. Schneider à adopter fréquemment les leçons du Parisinus et à modifier ainsi sensiblement le texte de Nicandre tel qu'il avait été connu jusque là et tel qu'il avait été édité en dernier lieu par J. G. Schneider.

Quasi un siècle après - quatre-vingt dix-sept ans, exactement - , fut publiée une nouvelle édition, due à deux spécialistes anglais de la littérature alexandrine, A. S. F. Gow et A. F. Scholfield. Quoiqu'elle fut accueillie très favorablement par la critique ${ }^{23}$, elle ne supplanta pas réellement celle d'O. Schneider ou, à tout le moins, ne réussit pas à s'en détacher et à la dépasser du point de vue de la méthode d'édition. Il est vrai que, dans l'introduction, les éditeurs déclarèrent n'avoir eu d'autre ambition que de fournir ce qu'ils appelèrent un first aid ${ }^{24}$, destiné à permettre au lecteur intéressé par le texte de Nicandre d'en prendre connaissance. Ainsi, reprirent-ils à leur prédécesseur allemand non seulement ses principes d'édition, comme il fut dit explicitement, mais aussi jusqu'à ses données relatives aux manuscrits, comme il apparut plus tard, même s'il y eut certaines nuances à l'égard des prises de position d'O. Schneider quant à l'évaluation de notre manuscrit. En effet, en ce qui concerne les principes d'édition, les deux anglais reprirent à Schneider son inventaire

21 O. Schneider 1856, p. 213.

22 Idem, pp. 215-216.

23 Voir, par exemple, Jacques 1955, p. 6, qui définit cette édition comme une oeuvre admirable et fait état du souci d'aborder de front les difficultés, de la rigueur d'un esprit qui ne se contente pas d'à peu près et d'honnêteté intellectuelle.

24 Gow \& Scholfield 1953, p. XI. 
des manuscrits, l'étude de leurs relations, quelque sommaire qu'ils l'aient considérée, et jusqu'à l'appréciation portée sur le texte du Parisinus ${ }^{25}$, qu'ils considérèrent comme «notablement différent» et, en général, «de loin supérieur» à celui de tous les manuscrits connus jusque là ${ }^{26}$. Quoique, par conséquent, ils lui firent la part belle dans l'édition du texte, ils notèrent que, au-delà de ses différences radicales et régulières par rapport au groupe formé par tous les autres manuscrits (qu'ils désignèrent par le sigle conventionnel $\Omega$ ), le $P a$ risinus (pourvu, lui, du sigle ח que lui avait attribué Schneider) descendait, en dernière analyse, d'un même modèle que les exemplaires du groupe $\Omega^{27}$. Ceci porta les deux anglais à réévaluer quelque peu le groupe de ces autres manuscrits par rapport à ce qu'en avait pensé Schneider, puisque, selon eux, il présentait la leçon correcte en plus de passages que ne l'avait soutenu l'allemand ${ }^{28}$; mais, simultanément, les éditeurs anglais considéraient que le texte de ces manuscrits avait subi l'inévitable usure que la copie manuelle imposait, volontairement ou non, aux ouvrages reproduits par des copistes «déconcertés» (sic) ${ }^{29}$, usure qui était d'autant plus marquée que, selon nos deux éditeurs, elle avait commencé très tôt pour le texte de Nicandre, avant même que ne se soient séparées ses deux versions $\Omega$ et $\Pi^{30}$.

Alors que, sur la trace d'O. Schneider, Gow et Scholfield reçurent dans le texte de leur édition tant de leçons du codex Parisinus et en citèrent un grand nombre dans l'apparat critique, il apparut très rapidement que, pas plus que leur prédécesseur, ils n'avaient consulté le manuscrit ni sur pièce ni même sur photo, mais avaient repris à l'apparat critique de l'allemand les leçons qu'ils en allégaient ${ }^{31}$. S'il en fallait encore une preuve, on pourrait citer les quelques exemples suivants de leçons mentionnées dans l'apparat de Gow et Scholfield,

25 Voir Idem, p. 10-11 pour l'inventaire des manuscrits, où ne sont mentionnés que les douze d'entre les vingt-deux inventoriés par Schneider qui furent utilisés par celui-ci dans l'établissement du texte, ainsi que cinq autres desquels Schneider reporta des leçons; pour les relations entre les manuscrits, voir p. 12 (voir, par exemple: «The relationships of the mss in the common class are complicated, and Schneider did little to elucidate them ... It might well prove difficult, and we do not here attempt, to relate the mss conclusively in a stemma codicum ...)».

26 Idem, p. 9.

27 Idem, p. 11.

28 Idem, p. 12.

29 Idem, p. 12: «bewildered scribes».

30 Idem, p. 12.

31 Voir Jacques 1955, p. 6: «... il (Gow) a repris essentiellement le texte d'O. Schneider en se bornant à puiser dans l'apparat touffu de son devancier un choix judicieux de variantes». 
qui ne figurent pas dans notre manuscrit, mais lui sont indûment attribuées dans l'apparat d'O. Schneider ${ }^{32}$ :

\begin{tabular}{|c|c|c|c|c|c|}
\hline $\mathrm{G} / \mathrm{S}$ & vers & texte & apparat & Parisinus & O.S. \\
\hline p. 30 & T. 35 & $\theta \imath \beta \rho \eta ́ s$ & $\theta u \mu \beta \rho \eta ́ s$ & $3,1.8$ & p. 35 \\
\hline p. 112 & A. 284 & $\tau^{\prime}$ & ö $\tau$ & $33 v, 1.6$ & p. 290 \\
\hline p. 115 & A. 320 & ${ }^{\mathcal{\varepsilon}} \nu \mu \hat{i} \xi \alpha 10$ & $\varepsilon{ }^{\prime} \mu \mu \hat{\prime} \xi \alpha 10$ & $35,1.4$ & p. 293 \\
\hline p. 128 & A. 511 & $\tilde{\omega}$ & $\tau \tilde{\omega}$ & $42 \mathrm{v}, 1.2$ & p. 304 \\
\hline & A. 517 & $\pi v \rho i ̀$ & $\varphi \lambda \circ \gamma i$ & $42 \mathrm{v}, 1.7$ & p. 304 \\
\hline
\end{tabular}

Il fallut attendre les travaux du français Jean-Marie Jacques pour que le Parisinus, cité pendant un siècle sans jamais avoir été consulté personnellement par les auteurs des travaux dont il fit l'objet, fût enfin collationné de façon rigoureuse par un traducteur et éditeur de Nicandre ${ }^{33}$. Peu après, l'italien Ignazio Cazzaniga s'attaqua lui aussi aux poèmes de Nicandre et consulta le Parisinus dont il déchiffra le texte sur l'original ${ }^{34}$. Il est vrai que, de Gow et Scholfield à Jacques et Cazzaniga, l'ecdotique subit une évolution plus forte que d'O. Schneider aux anglais; car, si après avoir identifié ses objet et méthode durant le XIX ${ }^{\text {ème }}$ siècle et être arrivée à un premier accomplissement dès l'époque de l'allemand, elle affina bien ses méthodes par la suite, elle ne les modifica cependant pas de façon substantielle jusqu'aux années cinquante de notre siècle. Ce n'est qu'alors qu'elle se transforma, notamment parce qu'elle intégra dans sa démarche les données de l'histoire des textes en plein développement, essentiellement par suite de l'introduction, dans le champ, de la paléographie et de la codicologie naissante, d'une part, et de la psychologie de l'acte de copie, de 1'autre ${ }^{35}$. C'est ainsi que Jacques et Cazzaniga - et

32 Dans la colonne $1(G / S)$, nous donnons la page de l'édition de Gow et Scholfield où figure le passage étudié; dans la 2 (vers), la référence du vers des Thêriaka $(=T$.) ou des Alexipharmaka (= A.); dans la 3 (texte), la leçon accueillie dans le texte par Gow et Scholfield, et à juste titre; dans la 4 (apparat), celle attribuée au Parisinus par l'apparat de Gow et Scholfield; dans la 5 (Parisinus), les références du feuillet du Parisinus où apparaît la leçon en question; dans la $6(O . S$.), la page de l'édition de Schneider dans l'apparat de laquelle figure la leçon prétendûment attestée par le Parisinus.

33 Voir Jacques 1955, avec, aux p. 7-8, les résultats de la collation pour les Alexipharmaka. Quant à l'édition, elle est celle de la thèse de doctorat mentionnée ci-dessus (note 3).

34 Voir les travaux de Cazzaniga 1956, 1957 et 1960, notamment.

35 Pour la paléographie et la codicologie, voir A. Dain, Les manuscrits, Paris, 1949 (2ème édition: Paris, 1964; 3ème édition: Paris, 1975), et, pour l'étude de l'acte de copie, voir G. Mogenet, Autolycus de Pitane. Histoire du texte suivie de l'édition critique des traités de la sphère en mouvement et des levers et couchers, (= Université Catholique de Louvain, Recueil des travaux d'histoire et de philologie, $3^{\text {ème }}$ série, $\mathrm{n}^{\circ}{ }^{\circ}$ 37), Louvain, 1950. 
plus l'italien que le français - purent porter sur le texte de notre manuscrit un regard singulièrement renouvelée ${ }^{36}$.

Cazzaniga, qui préparait une nouvelle édition critique de nos deux poèmes ${ }^{37}$ et qui, dans ce cadre, avait encouragé ses disciples à en éditer, comme en préalable ${ }^{38}$, les paraphrases d'Euteknios (d'époque inconnue, mais à situer entre les II ${ }^{\text {ème} / I I I ~}{ }^{2}$ me siècles et le début du VI $\left.{ }^{\text {ème }}\right)^{39}$ et les scolies médiévales, identifia ainsi dans le texte de notre manuscrit l'intervention d'un réviseur ${ }^{40}$. Avec un sens de la paléographie qui n'avait d'égal que son acribie philologique, il effectua de brillantes corrections qui lui permirent, simultanément, d'identifier des mécanismes d'erreur qui avaient conduit aux leçons fautives présentes dans les manuscrits, que soit celui de Paris ou ceux du groupe $\Omega$. Ainsi, débusqua-t-il à travers le texte de notre codex une main qui réagissait à l'égard de son modèle là où celui-ci était ou lui paraissait détérioré; elle substitua de la sorte des gloses à ces leçons inexactes, mais sans retrouver nécessairement le texte susceptible d'avoir été celui de Nicandre; plus même: dans certains cas, ce réviseur ferait preuve, selon Cazzaniga, d'une certaine gaucherie. Constatant, par ailleurs, que le texte du Parisinus présente des leçons divergentes là où celui des autres exemplaires est manifestement corrompu, Cazzaniga en déduisit que le modèle au départ duquel travailla notre correcteur était sinon identique, du moins fortement semblable à celui de ces autres manuscrits, et que, précisément, les interventions du correcteur visaient à tenter de restaurer la teneur originale du texte sur ces points ${ }^{41}$.

La conclusion majeure du travail de Cazzaniga fut, dans la perspective qui est la nôtre ici, que le texte du Parisinus avait été surévalué par O. Schneider et ne devait pas nécessairement être préféré à celui des exemplaires du groupe $\Omega$, lequel, au contraire, permet, dans plus d'un cas, d'expliquer la genèse

36 Voir notamment Cazzaniga 1960/2, 1963 et 1973.

37 Sur ce point, voir Touwaide 1991, p. 14.

38 Voir, en ordre chronologique de publication: Gualandri 1968 (paraphrase des Thêriaka); Crugnola 1971 (scolies des Therriaka); Gualandri 1974 (scolies des Alexipharmaka); Geymonat 1976 (paraphrase des Alexipharmaka). Dans le même temps et de façon indépendante, les deux paraphrases furent aussi éditées par Papathomopoulos 1976, qui vient, par ailleurs, de publier une concordance intégrale du texte de Nicandre (Papathomopoulos 1996).

39 Sur Euteknios, voir: H. Gärtner, Euteknios, dans Der Kleine Pauly, vol. 2, Munich, 1967, col. 465. Les repères chronologiques sont ceux d'Oppien, dont nous possédons une paraphrase attribuée à Euteknios, et du Vindobonensis medicus graecus 1, des environs de 512, le plus ancien manuscrit qui contient le texte des paraphrases d'Euteknios.

40 Cazzaniga 1963/2, pp. 185, 195 et 197-198.

+1 Cazzaniga 1960/2, passim. 
des leçons apparemment meilleures du Parisinus et avait, de ce fait, quelque chance d'être plus proche de la lettre de l'original.

Ce travail de rééquilibrage fut confirmé et approfondi par la suite, et de façon indépendante par rapport à Cazzaniga, par l'anglais White, auteur d'un commentaire des Idylles de Théocrite et d'une étude sur la poésie alexandrine $^{42}$. En effet, à partir de l'analyse philologique d'une quarantaine de passages des deux poèmes de Nicandre, il montra, d'une part, que le témoignage du groupe de manuscrits $\Omega$ devait être préféré à plusieurs leçons du Parisinus, au contraire de ce qu'avaient fait Gow et Scholfield, et, d'autre part, que des corrections d'éditeurs introduites dans le texte de nos deux poèmes par $O$. Schneider et, à sa suite, par Gow et Scholfield, ne se justifiaient pas, le texte attesté par les manuscrits pouvant donner toute sa cohérence aux passages étudiés, et ceci sans avoir procédé à une collation de notre codex, mais au départ de l'apparat critique de l'édition de Gow et Scholfield. Ci-dessous, nous reprenons de façon synthétique les résultats du travail de White ${ }^{43}$ :

$\begin{array}{llll}\text { Thêriaka } & 27 & 7-8 & \text { codd. } \\ & 29 & 8-9 & \text { codd. } \\ & 30 & 9 & \text { cett. }(\text { id } \text { est: } \text { non } B \text { et } P \text { ) } \\ 46 & 9-11 & \text { codd. } \\ & 165 & 15-16 & \Omega \\ 172 & 15-16 & \text { B } \\ 268 & 17-19 & \text { GLM } \\ 283 & 19 & \Omega \\ 291 & 19-21 & \text { codd. } \\ 292 & 19-21 & \text { codd. } \\ 342 & 23-24 & \Omega \\ 385 & 24-25 & \Omega \\ 446 & 26-27 & \text { codd. } \\ 562 & 33-35 & \text { codd. } \\ 628 & 40-41 & \text { codd. }\end{array}$

42 White 1987.

43 Nous mentionnons successivement les vers des poèmes de Nicandre étudiés par White, les pages de son travail où ils sont traités et la source à suivre selon l'anglais pour éditer ces vers, différente de celle suivie par Gow et Scholfield et identifiée selon les sigles suivants: codd. pour codices, désignant les manuscrits unanimes; cett. pour ceteri, c'est-àdire tous les manuscrits à l'exception de ceux mentionnés; - B: Leidensis Vossianus graecus Q 39; - G: Goettingensis phil. 29; - L: Lorrianus (manuscrit non identifié actuellement ou disparu); - M: Florentinus Laurentianus 32.16; - P: Parisinus graecus 2728; -R: Florentinus Riccardianus 56; - V: Venetus Marcianus graecus 480; - $\Omega$ : l'ensemble des manuscrits autres que le Parisinus, unanimes et opposés à ce dernier. 


$\begin{array}{llll}642 & 42-44 & \text { codd. } \\ 820 & 55-57 & \Omega \\ 824 & 57-60 & \Omega \\ 870 & 61-63 & \text { codd. } \\ 870 & 61-63 & \text { codd. } \\ 895 & 63-64 & \text { codd. } \\ 927 & 64-65 & \text { codd. } \\ & 944 & 65-66 & \text { codd. } \\ & 25 & 73-74 & \text { GMRV } \\ 42 & 74-76 & \text { codd. } \\ 92 & 78-79 & \text { cett. (id est: non } G M V) \\ 99 & 79-80 & \text { codd. } \\ 277 & 89-91 & \Omega \\ 278 & 89-91 & \Omega \\ 396 & 92-93 & \Omega \\ 423 & 95-96 & \Omega \\ 454 & 97-98 & \text { codd. } \\ 463 & 99-100 & \Omega \\ 464 & 99-100 & \Omega \\ 472 & 101-103 & \Omega \\ 483 & 103-104 & \text { cett. (id est: non } G) \\ 491 & 104-105 & \text { cett. (id est: non } G) \\ 499 & 106-108 & \text { codd. } \\ 538 & 110-112 & \text { codd. } \\ & & \end{array}$

Lorsque nous avons entrepris l'examen du Parisinus, c'était là le travail le plus récent sur la question; il s'inscrivait dans le cadre d'une lente évolution, tendant à revoir le rôle que le Parisinus était appelé à jouer dans l'établissement du texte de Nicandre.

\section{Nouvelles perspectives pour l'édition du texte de Nicandre.}

Notre analyse du Parisinus et de l'histoire du texte que suggèrent ses accidents ${ }^{44}$, nous a conduit, sur les pas de Cazzaniga, à proposer une modification significative de l'attitude à prendre à l'égard du manuscrit parisien dans l'éta-

44 Cette notion d'accident, reprise à J. Mogenet (cité à la note 35), s'applique à toute particularité textuelle d'un manuscrit, relevée en fonction d'un exemplaire de collation choisi arbitrairement. Les résultats de cette analyse, avec l'histoire du texte à laquelle ils portent, seront très probablement publiés dans la Byzantinische Zeitschrift, sans doute dans le volume de 1998 . 
blissement du texte de Nicandre. En effet, alors qu'O. Schneider, puis Gow et Scholfield accordaient, comme nous l'avons vu, une prééminence au Parisinus, parce que, pensaient-ils, il offrait une version du texte de Nicandre supérieure à celle des manuscrits de la famille $\Omega$, il nous est apparu que le texte du Parisinus est composite et constitue une sorte de méta-texte, fait non seulement de paradiorthoses, mais aussi de gloses et explications venues se substituer au texte original. Sans doute est-cela qui provoqua l'impression d'une supériorité du texte du Parisinus, effectivement plus clair en de certains passages, et qui donna l'impression de pouvoir introduire dans l'édition de Nicandre une rupture avec la pratique antérieure, et d'autant plus que l'ecdotique de l'époque d'O. Schneider fut souvent conquérante, croyant retrouver avec hardiesse les vestiges et monuments littéraires de la Grèce antique, en une vision dont on perçoit aujourd'hui combien elle fut romantique ${ }^{45}$.

Ainsi, ni O. Schneider ni les éditeurs anglais ne semblent avoir noté que le vers 171 des Thêriaka absent du Parisinus pourrait avoir été omis par saut du même au même. En effet, le vers 170 se termine par une finale en - $\tau \omega \mathrm{v}$ comme le vers 171 , laquelle aurait pu avoir provoqué le saut des yeux d'un copiste dans le modèle qu'il était occupé à reproduire, selon un mécanisme qui est loin d'être rare dans la transmission des textes. Dès lors, il ne convient sans doute pas de considérer, comme le font la scolie ${ }^{46}$ et Cazzaniga ${ }^{47}$, que le vers a été athétisé parce qu'il aurait paru suspect au rédacteur du texte $\Pi$, pas plus - et encore moins - qu'il ne fut absent du Parisinus parce qu'il n'aurait pas appartenu au texte de Nicandre! Par conséquent, on admettra ce vers dans le poème, sans plus s'interroger désormais sur son authenticité.

Parmi les nombreux cas où il ne faudra désormais plus suivre le Parisinus, nous relèverons ici les suivants, significatifs du travail de révision dont le texte du manuscrit parisien a fait l'objet ${ }^{48}$ :

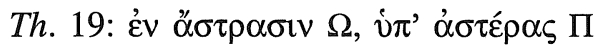

Le passage, qui raconte le catastérisme d'Orion, se conclut par le vers 19, avec cette affirmation qu'Orion est fixé parmi les astres. Il semble qu'il faille préférer

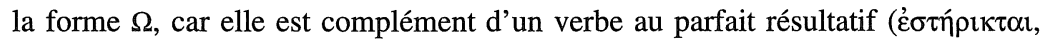

45 Sur cette question, voir D. Gourevitch (éd.), Médecins érudits de Coray à Sigerist. Actes du colloque de Saint-Julien-en-Beaujolais (juin 1994) (= Collection De l'archéologie à l'histoire), Paris, 1995, avec une analyse des méthodes d'édition et d'étude des textes médicaux durant le XIX ${ }^{\text {ème }}$ siècle essentiellement.

46 Crugnola 1971, p. 95, sub 171 a.

47 Cazzaniga 1973, p. 79.

48 Nous citons ces cas selon leur ordre d'apparition dans les poèmes (et non selon, par exemple, les mécanismes de déformation dont ils procèdent), commençant par les Thêriaka. 
vers 20), qui favorise plus un datif complément de situation comme l'est la leçon $\Omega$, qu'un accusatif complément de mouvement comme la leçon $\Pi$. Cette conclusion est confirmée par la scolie ${ }^{49}$, où l'on voit apparaître la leçon de $\Omega$ (avec une variante lexicale, mais au datif), ainsi que par la paraphrase d'Euteknios, qui donne un complément prépositionnel avec c̉v et le datif ${ }^{50}$.

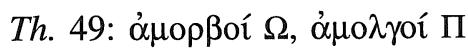

Le manuscrit parisien présente une leçon résultant sans doute d'une correction. Après avoir mentionné la pierre de Thrace (vers 45-47), Nicandre précise, en effet, qu'elle se trouve auprès d'un fleuve fréquenté par des Thraciens (vers 48-50), désignés par le terme qui constitue la leçon étudiée ici. Il est clair qu'il

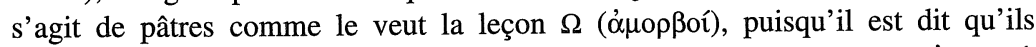

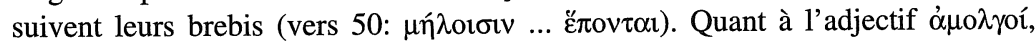
interprété dans la bibliographie comme signifiant 'qui boit du lait' ou 'qui trait le lait' ${ }^{51}$, il vise probablement à décrire le régime alimentaire de ces Thraciens,

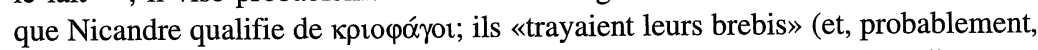
en buvaient le lait) et «mangeaient la chair de leurs bêliers». Cependant, l'on note que la confusion $\lambda / \rho$ est fréquente dans le texte du manuscrit parisien et pourrait s'être produite dans la leçon ó $\mu_{0} \rho \beta$ oí, donnant ainsi lieu à un incompréhensible

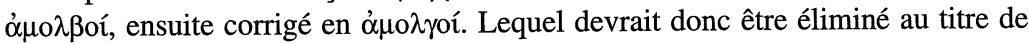
paradiorthose, quelque vraisemblable que soit le tableau créé par cette allusion à la vie des pâtres thraciens.

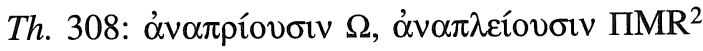

Dans un tableau clinique, Nicandre évoque, au moyen du verbe dont il est question ici, un symptôme dont sont affectées les dents. La leçon de $\Omega$, au sens incertain ('scier de bas en haut' [?]), est de toute évidence inadéquate; quant à celle du Parisinus, qui n'apparaît ni chez Euteknios ni dans la scolie ${ }^{52}$, elle appartient au langage médical, où $\alpha v \alpha \pi \lambda \varepsilon^{i} \omega$ était le verbe propre pour désigner le fait de 'perdre des dents' ${ }^{53}$; il y a donc quelque probabilité pour qu'elle résulte d'une intervention, au départ d'un texte corrompu, sans doute identique à celui que nous connaissons actuellement dans le groupe $\Omega$.

Cazzaniga a suggéré de corriger la leçon de $\Omega$ en $\alpha^{\prime} v \alpha \beta$ pítovouv ${ }^{54}$, en se référant notamment aux vers 209-210 des Alexipharmaka où apparaît le verbe

49 P. 43, sub 19 e et f, éd. Crugnola.

50 P. 4, 1. 21-22, éd. Papathomopoulos.

51 Voir, respectivement, Gow 1951, p. 98, et Crugnola 1962, p. 104-141, le second sens étant probablement plus exact.

52 Pour Euteknios, voir p. 37, 1. 4-5 de l'édition de Gualandri 1968 et, pour la scolie, p. 138 de l' édition de Crugnola 1971.

53 Voir dans le lexique hippocratique de H. J. Kühn \& U. Fleischer, Index Hippocraticus. Cui elaborando interfuerunt sodales Thesauri Linguae Graecae Hamburgensis ..., Göttingen, 1986-1989, p. 49, sub uerbo, avec (ibidem) la variante $\alpha \dot{v} \alpha \pi \lambda \omega \omega$, appliquée aux os qui, brisés, remontent à la surface, provoquant des fractures ouvertes.

54 Cazzaniga 1960/2, pp. 181-183. 
$\beta p i ́ \theta \omega$, accompagné de celui qui signifie 'être pesant'. Or, la leçon proposée par l'italien présente l'avantage de pouvoir expliquer l'origine de celle de $\Omega$ par une confusion auditive entre $\pi$ et $\beta$ et une confusion entre un $O$ et un $\Theta$ majuscules, et a donc quelque chance d'être juste.

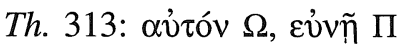

Le pronom du texte $\Omega$ est certes banal, mais le substantif de $\Pi$ (dans sa couche) semble être une précision additive et explicative. En effet, les vers 312-315 narrent l'accident subi en Egypte par Thon, le pilote du navire d'Hélène: lorsqu'il

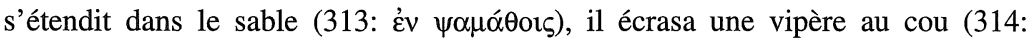

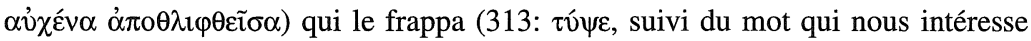
ici) ${ }^{55}$. Il faut relever que la scolie confirme le pronom de $\Omega^{56}$, tandis que la paraphrase d'Euteknios est trop vague en ce passage pour être de quelque utilité que ce soit ${ }^{57}$.

\section{Th. 631: omisit Parisinus}

Le cas de ce vers pourrait n'être pas dissemblable de celui du vers 171 des Thêriaka présenté ci-dessus, et d'autant moins que, dans le Parisinus, le vers 630 s'achève sur la finale - HN, par suite d'une erreur de majuscule due à la transformation en un $\mathrm{N}$ du iota adscrit. Or, cette finale - HN pourrait avoir été transformée en -IN par itacisme, soit la séquence suivante: $-\mathrm{HI} \rightarrow-\mathrm{HN} \rightarrow-\mathrm{IN}$

Car, c'est précisément sur une telle finale que s'achève le vers 631 omis. Dès lors, il aurait pu y avoir saut de la finale du vers 630 à celle du vers 631 et, par conséquent, omission du vers 631. Lequel ne serait donc pas à considérer comme athétisé par le Parisinus, et d'autant moins qu'il appartint au texte à partir duquel furent établies la paraphrase d'Euteknios et la scolie ${ }^{58}$.

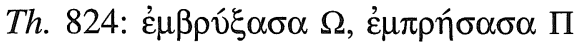

Dans la description de la murène et de la résistance qu'elle oppose aux pêcheurs qui tentent de la prendre (vers 822-825), apparaît ce participe, appliqué au poisson. Il semble préférable d'adopter $\varepsilon \mu \beta \rho v ́ \xi \alpha \sigma \alpha$ ('mordre'), au lieu de $\varepsilon \dot{\varepsilon} \pi \rho \eta ́ \sigma \alpha \sigma \alpha$ ('s'enflammer') présent dans le Parisinus: cela justifierait à tout le moins l'affirmation des vers 823-825 selon laquelle, la murène, s'étant échappée du réservoir à poissons, suscite la panique des pêcheurs qui, terrorisés, se jettent à l'eau ${ }^{59}$. L'on y sera d'autant plus porté que le terme apparaît en deux autres

55 Il convient de noter que le terme ev̉ví apparaît au vers 55 des Thêriaka dans la

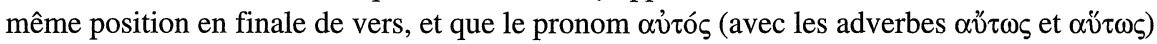
figure aussi en finale de vers à plusieurs reprises dans les Thêriaka $(19,278,444,723$, $879,910)$.

56 P. 140, sub 313 b, éd. Crugnola.

57 P. 19, 1. 1-17, éd. Papathomopoulos.

58 P. 36, 1. 5-6, éd. Papathomopoulos, pour la paraphrase d'Euteknios, et 236, sub 631, éd. Crugnola, pour la scolie.

59 Dans le même sens, voir White 1987 , p. 60, note 83. 
passages des poèmes de Nicandre, où il occupe la même position métrique dans le vers ${ }^{60}$. Enfin, on notera que la scolie, qui ne glose pas le participe de façon spécifique, mais paraphrase l'ensemble du passage, donne comme équivalent $\delta \alpha-$

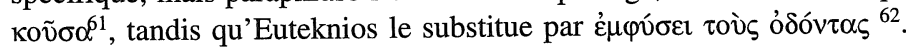

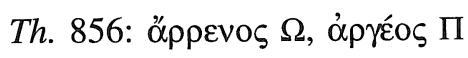

L'adjectif est appliqué à la fleur de la plante $\varphi \lambda{ }^{\prime} \mu \varsigma_{\text {, }}$ qui entre en composition dans la préparation d'un remède. Pour clarifier ce passage, l'on dispose du Traité de matière médicale de Dioscoride, où la plante est décrite ${ }^{63}$; l'on y apprend ainsi qu'elle présente deux espèces, l'une dite noire et l'autre blanche, et que cette dernière se subdivise encore en mâle et femelle. Par ailleurs, lorsqu'il doit citer les différentes espèces et sous-espèces, Dioscoride mentionne ainsi la noire, la femelle et la mâle, sans mentionner la blanche. Il semble que la leçon öppevos de $\Omega$ corresponde plus à l'usage de la pharmacologie, même si l'expression qui résulterait de la leçon de $\Pi$ se retrouve dans les Alexipharmaka, au vers 305. Notons, enfin, que la leçon de $\Pi$ apparaît dans la scolie au titre de variante ( $\gamma \rho \alpha ́ \varphi \varepsilon \tau \alpha \mathrm{l}$ ) de celle de $\Omega$ donnée en lemme ${ }^{64}$, tandis que Euteknios ne suit pas le texte d'assez près pour permettre de confirmer notre choix ${ }^{65}$.

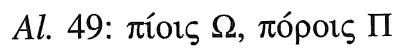

Dans cette prescription, la leçon de $\Omega$ ( $\pi$ ív $\omega$, 'boire', qui devrait être utilisé ici dans un sens factitif, 'faire boire'), pour banal qu'elle soit, l'est cependant moins que celle de П ( $\pi$ ó $\omega$, 'procurer', soit, en contexte thérapeutique, comme c'est le cas ici, 'administrer'). Les deux verbes sont abondamment utilisés par Nicandre 66. $\pi i v \omega$ l'est à seize reprises, mais pas dans le passage relatif au traitement de l'empoisonnement dû à l'aconit, tandis que $\pi$ óp $\omega$ l'est en vingt occasions, dont deux dans ce passage (vers 68 et 71). Sans doute faut-il préférer, dès lors, la leçon de $\Omega$.

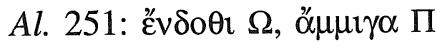

L'adverbe, utilisé par Nicandre dans le tableau des symptômes provoqués par l'absorption de la boisson toxique faite de colchique, apparaît dans la description de l'apparition d'une sensation de picotement sur les lèvres. Celui de $\Omega$ est attesté dans la poésie homérique et hésiodique essentiellement ${ }^{67}$ et est confirmé par la

60 Th. 271, et Al. 338.

61 P. 290, 1. 13 éd. Crugnola.

62 P. 45, 1. 16-17 éd. Papathomopoulos.

63 IV, 103 (= vol. 2, p. 257-259 éd. M. Wellmann, Pedanii Dioscuridis Anazarbei, De materia medica liri quinque, éd. -, 3 vol., Berlin, 1906-1914 [réimpression: Berlin, 1958]).

64 P. 298, sub 856 c, éd. Crugnola.

65 P. 47, 1. 6-8, éd. Papathomopoulos.

66 Voir O. Schneider 1856, notre index, Berkowitz 1980 et Papathomopoulos 1996, sub uerbis.

67 Pour les occurrences des termes étudiés ici, nous nous fondons des dictionnaires de 
paraphrase d'Euteknios ${ }^{68}$; par ailleurs, il est utilisé par Nicandre en trois autres passages du même poème ${ }^{69}$, où il figure toujours dans la même position dans le vers que dans le présent passage et présente un sens qui peut être celui requis ici: 'à l'intérieur', signifiant par là que le phénomène décrit ne porte pas sur la superficie des muqueuses, mais agit en profondeur. La leçon de $\Pi$ se retrouve dans dix passages de Nicandre ${ }^{70}$, n'y occupant qu'à deux reprises une position à l'intérieur du vers identique à celle qu'elle aurait ici ${ }^{71}$; elle semble par ailleurs avoir été utilisée à une époque plus récente et pourrait être une banalisation du premier adverbe, et d'autant plus que son sens s'adapte moins bien au présent passage.

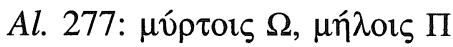

Dans cette énumération des ingrédients d'un médicament, la leçon de $\Omega$ ('des baies de myrte'), confirmée par la scolie et la paraphrase d'Euteknios ${ }^{72}$, l'est aussi par les traités de toxicologie ${ }^{73}$, au contraire de celle de $\Pi$ ('des pommes'). Cette dernière pourrait résulter d'une tentative de correction sur la leçon de $\Omega$ déformée par suite d'une faute d'orthographe (itacisme qui aurait provoqué la modification de $-v-$ en $-\eta-)$ et de la confusion fréquente, dans le texte $\Pi$, entre $-\lambda$ - et $-\rho-$.

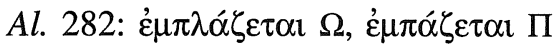

Le leçon du Parisinus ne convient manifestement pas, puisqu'elle signifie 's'attacher à', alors que celle de $\Omega$, qui se traduit par 'être égaré', est bien adaptée au présent passage où il est question de folie et, dans le présent hémistiche, d'accélération du rythme cardiaque ${ }^{74}$. Sans aucun doute, la leçon de $\Pi$ est-elle

langue grecque, non sans en avoir vérifié les informations sur les textes eux-mêmes, soit le Liddell-Scott-Jones et le dictionnaire espagnol édité sous la direction de F. Rodríguez Adrados (5 volumes parus); de même, pour la sémantique, nous avons consulté plus spécialement les dictionnaires étymologiques de Frisk et de Chantraine. Sauf exceptions, nous avons omis de donner les références à ces dictionnaires autant qu'aux textes eux-mêmes, afin de ne pas alourdir le présent article.

68 P. 68, 1. 21, éd. Papathomopoulos.

69 Outre O. Schneider 1856 et notre index, voir Berkowitz et Papathomopoulos, sub uerbo: Al. 192, 262, 316.

70 Voir O. Schneider 1856, notre index, Berkowitz et Papathomopoulos, sub uerbo.

71 Th. 941, et $\mathrm{Al} .548$.

72 Voir p. 114, sub 277 a, éd. Geymonat pour la scolie, et p. 69, 1. 20, éd. Papathomopoulos pour la paraphrase.

73 Voir, par exemple: Pseudo-Dioscoride, Alexipharmaka, chap. 5 (= p. Al. XX, 1. 12, éd. A. Touwaide, Les deux traités de toxicologie attribués à Dioscoride. La tradition manuscrite grecque. Edition critique du texte grec et traduction, 5 vol., Louvain, thèse dactylographiée [= p. 20,1.12, éd. K. Sprengel, Leipzig, 1830]).

$74 \mathrm{La}$ leçon de $\Omega$ est confirmée par la scolie (p. 115, sub 282 b, éd. GEYMONAT), sans l'être par la paraphrase d'Euteknios, trop imprécise sur ce point (p. 70, 1. 2-5, éd. Papathomopoulos). 
apparue par simplification des lettres $\Lambda$ et A dans la graphie majuscule. Quoique la leçon de $\Omega$ soit satisfaisante, Jacques a suggéré de la corriger en é $\mu \pi \alpha ́ \lambda \lambda \varepsilon \tau \tau \propto \imath$ ('s'élancer') ${ }^{75}$, sans que cela soit nécessaire, nous semble-t-il.

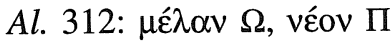

La qualification de 'noir' attribuée par $\Omega$ au toxique qu'était censé être le sang de taureau ${ }^{76}$, aura certes de quoi surprendre, tandis que celle de $\Pi$ ('frais') correspond à celle attribuée à cette substance par les traités de toxicologie ${ }^{77}$. Cependant, en raison de sa technicité même, cette leçon de $\Pi$ semble pouvoir être une addition par rapport à celle de $\Omega$, et d'autant plus que cette dernière correspond à des usages homériques perpétués par la tragédie, dans lesquels l'adjectif noir est utilisé pour définir la mort, la souffrance, voire le sort.

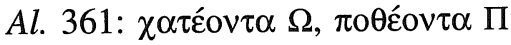

Les deux participes, intervenant dans la description d'une thérapie à administrer à un patient malgré lui, ont une même signification ('désirer'); cependant, le premier est typiquement homérique et apparaît ici dans une même formule que dans une de ses attestations de l'Iliade ${ }^{78}$, alors que le second appartient à la langue courante et doit donc probablement être considéré comme une glose de l'autre.

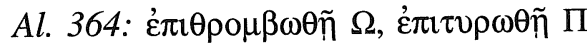

Le passage où apparaît ce participe traite du lait caillé dans l'estomac, jadis considéré comme toxique. Celui de $\Omega$ semble avoir un sens impropre ('qui est coagulé'), tandis que celui de $\Pi$ présente un sens exact ('qui devient du fromage'). Les choses sont cependant moins claires, car, outre que la leçon de $\Pi$ correspond à l'une des gloses fournies par la scolie ${ }^{79}$, celle de $\Omega$ reprend le terme utilisé dans les traités techniques pour qualifier le sang de taureau auquel est toujours associé le lait caillé dans l'estomac ${ }^{80}$. Il faut admettre une certaine élasticité dans l'usage, car, d'une part, Nicandre utilise le terme $\theta$ pó $\mu ß \varsigma_{\text {( }}$ ('caillot'), avec ses variantes morphologiques, dans des sens qui ne sont pas nécessairement le leur, de façon exacte, notamment pour désigner les oeufs de poule dépourvus de co-

75 Jacques 1955, p. 21, note ad v. 282.

76 Sur ce point, voir A. Touwaide, «Le sang de taureau», L'Antiquité Classique, 48 1979), p. 5-14.

77 Voir par exemple Pseudo-Dioscoride, Alexipharmaka, chap. 25 (= p. Al. XXXIV, 1. 10, éd. Touwaide [citée à la note 73] [= p. 34, 1. 10, éd. K. Sprengel, Leipzig, 1860]). Il faut noter que ni la scolie ni la paraphrase d'Euteknios ne se sont arrêtées sur ce terme.

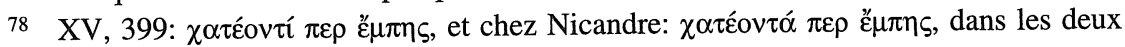
cas en finale de vers.

79 p. 135, sub 364 a, éd. Geymonat.

80 Voir, entre autres: Pseudo-Dioscoride, Alexipharmaka, chap. 26 (= p. Al. XXXV, 1.

$11 \& 12$, éd. Touwaide [= p. 35, 1. $11 \& 12$, éd. K. Sprengel, Leipzig, 1830]). 


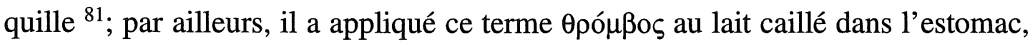
au vers 373 , où il précise que la présure peut dissoudre les caillots. Si l'on accepte la leçon de $\Omega$ dans le vers 364 ( $\dot{\varepsilon} \pi \theta \rho \circ \mu \beta \omega \theta \tilde{n})$, il pourrait y avoir là un subtil rappel de ce qui est constitutif du tableau clinique de ce toxique supposé, et, simultanément, une insistance sur l'efficacité de la substance thérapeutique pres-

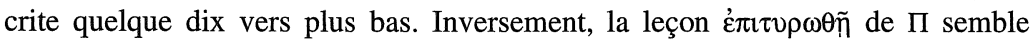
relever d'un souci plus exclusivement linguistique d'explicitation de l'effet poétique qu'implique le terme attesté par $\Omega$ et serait une précision additive, peut-être portée dans un premier temps dans la marge ou entre les lignes du texte et introduite par la suite dans ce dernier.

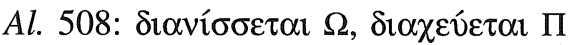

Les deux verbes sont appliqués au souffle qui passe par la gorge lors de l'absorption de sangsues; ils sont presque équivalents d'un point de vue sémantique, puisque le premier signifie 'passer à travers' et le second 'couler à travers' Alors que celui de $\Pi$ appartient à la langue courante, celui de $\Omega$ est rare, n'apparaissant, avant Nicandre, que chez Pindare ${ }^{82}$; il pourrait avoir été choisi pour ce motif, tandis que l'autre serait une glose.

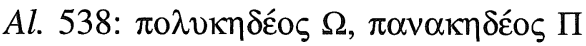

L'adjectif détermine la salamandre, créditée de propriétés toxiques durant l'Antiquité et apparaissant pour cette raison dans le poème de Nicandre (vers 537-

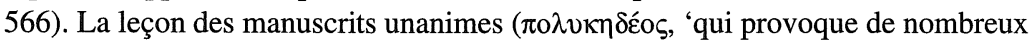
maux') correspond bien à la suite du texte où sont décrits tous les troubles consécutifs à l'absorption d'une boisson empoisonnée avec de la salamandre. Celle

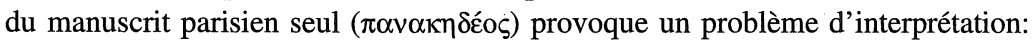
traduite par le Liddell-Scott-Jones par 'free from all care' ('totalement insouciante'), elle est peut-être à rapprocher plutôt de l'adjectif $\pi \alpha v \alpha \kappa n ́ \varsigma$ et du substantif $\pi \alpha ́ v \alpha \kappa \varepsilon \varsigma$ (soit, respectivement, 'qui traite tous les maux' et la plante appelée panakes, traditionnellement identifiée à une férule). Cependant, ni l'un ni l'autre des deux sens ne convient, quelle que soit l'étymologie que l'on attribue à l'adjectif, de telle sorte qu'il faut sans doute éliminer la leçon de $\Pi$ au profit de celle de $\Omega$ et considérer la première comme un accident de tradition manuscrite ${ }^{83}$.

Cet examen philologique, effectué notamment dans le cadre de la poétique de Nicandre, met bien en évidence le travail d'élucidation opéré dans le texte $\Pi$, dont on perçoit mieux ainsi la gamme variée des composantes, avec autant des retours à la langue courante que des emprunts à la langue technique, mais aussi des faux rapprochements, des erreurs et des néologismes fallacieux. Dans

81 Al. 295.

82 Pyth., XII 25 (= p. 169, éd. A. Puech, Paris, 1922).

83 Dans le même sens, voir Touwaide 1977, p. 35, et White 1987, p. 111-112. 
l'édition du texte de Nicandre, il faudra désormais se garder de trop se référer au Parisinus, puisque, comme nous espérons l'avoir montré ici, il est contaminé, en quelque sorte, par les résultats d'une lecture, avec des gloses, explications et autres interventions interprétatives; de la sorte, il constitue un métatexte plus qu'il ne transmet la lettre originale des poèmes de Nicandre.

Ceci ne signifie pas pour autant que le texte $\Pi$ soit dépourvu d'intérêt pour l'éditeur, puisque, par sa proximité avec le stade de la graphie majuscule et par le conservatisme scrupuleux avec lequel il a été reproduit, il a préservé mieux que le texte $\Omega$ du matériel ancien, dont il est sans importance qu'il soit fautif. Car, à travers celui-ci, une analyse philologique dûment conduite, notamment avec l'aide de la paléographie, peut arriver à retrouver des leçons sans doute originales, qui permettent à tout le moins d'expliquer la génétique des leçons erronées du texte $\Pi$ autant que $\Omega$, et représentent donc, sinon la forme originale du texte, du moins une version antérieure à celles des textes $\Pi$ et $\Omega$, comme l'a brillamment démontré Cazzaniga ${ }^{84}$.

Ainsi, parmi les leçons probablement authentiques qu'a conservées le Parisinus, citera-t-on les trois cas suivants:

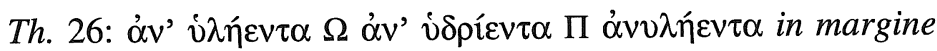

Evoquant les biotopes des serpents, Nicandre cite ainsi les collines (avec un singulier à valeur de collectif), déterminées par l'adjectif examiné ici. La leçon de $\Omega$ ('non boisé', si l'on considère qu'il y a eu mécoupure du terme, par suite d'une mélecture du texte dans la graphie majuscule) rappelle le vers $147 \mathrm{du}$ poème, où Nicandre décrit le biotope d'un serpent (avec le terme ìńَ $\varepsilon$ ), et est confirmée par la scolie ${ }^{85}$; elle ne l'est pas, par contre, par Euteknios, qui parle «d'endroits où court de l'eau ( $(\because \delta \omega \rho)$, tels les collines, les bois et les prés humides, qui constituent la pâture des animaux» ${ }^{86}$. Cette mention de l'eau chez Euteknios tenderait à accréditer la leçon de $\Pi$, pour autant que l'on en corrige la faute d'orthographe, avec le - - - provenant d'un - $\eta$ - par itacisme; le mot ainsi obtenu ( $\delta \delta \rho \eta ́ \varepsilon v \tau \alpha)$ signifie, précisément, 'arrosé'; dans ce cas, le $\alpha v^{\prime}$ ' serait une préposition en tous points correcte.

Or, si l'on pose que la leçon ainsi reconstituée à partir de celle de $\Pi$ dût être l'originale, on peut arriver à celle de $\Omega$, à la suite d'une erreur de majuscule, avec le passage de $-\Delta$ - à $-\Lambda$-; puis, la séquence $-\Lambda \mathrm{P}$ - ainsi obtenue aurait été simplifiée, en raison de son incompatibilité avec la phonétique; soit la séquence suivante de déformations cumulées: Y $\triangle$ PHENTA > YAPHENTA > Y $\Lambda$ HENTA.

Dans ce cas, il reste que cette dernière leçon a été portée dans la marge de $\Pi$, comme si elle était destinée à corriger celle présente dans le texte, qui, de ce fait,

84 Voir Cazzaniga 1960/2.

85 Crugnola 1971, p. 45.

86 P. 5, 1. 15-16, éd. Papathomopoulos. 
semble erronée. Mais il s'agit moins d'une correction que d'une report sur le texte $\Pi$ des leçons variantes du texte $\Omega{ }^{87}$.

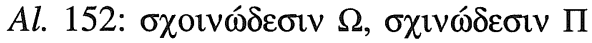

Dans l'énumération des médicaments destinés à traiter l'empoisonnement dû à la cantharide, Nicandre cite la terre de Samos (vers 148-149), qui lui fournit le point de départ du tableau géographique (vers 149-152) dans lequel intervient l'adjectif étudié ici. Celui-ci est appliqué aux flancs d'un des sommets de l'île,

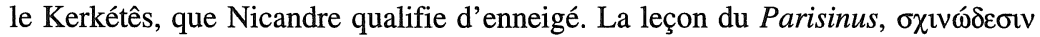
('qui a des lentisques'), semble devoir être acceptée contre celle de $\Omega$, $\sigma \chi 01 v \omega ́ \delta \varepsilon-$ ouv ('qui a des joncs'), non seulement parce que la seconde leçon s'explique aisément à partir de la première par l'itacisme, mais aussi, voire surtout, parce que la première permet d'évoquer le biotope des îles égéennes avec un élément qui en est caractéristique, tandis que la seconde aboutit sinon à un non-sens, du moins à une information étrange ${ }^{88}$.

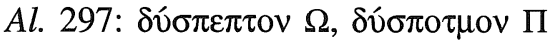

Les leçons attestées par les deux textes sont théoriquement possibles d'un point de vue lexical et s'intègrent toutes deux bien dans la syntaxe de la phrase; celle de $\Omega$ signifie 'difficile à digérer' et celle de $\Pi$ 'qui a un destin difficile'. Par ailleurs, la scolie confirme celle de $\Omega{ }^{89}$, tandis qu'Euteknios n'a pas adhéré d'assez près au texte grec en ce passage pour fournir quelque leçon que ce soit ${ }^{90}$. Il ne reste qu'à recourir au sens des vers pour tenter de choisir entre l'une et l'autre leçon. Nicandre y effectue une comparaison entre des déjections d'un patient intoxiqué par le chardon à glu et des oeufs d'une poule qui a été mise à mal: ils ont été pondus sans coquille, dans la peau sous-jacente à la coquille, et constituent, pour la poule, une descendance qualifiée par l'adjectif dont nous tentons d'identifier ici quel il devrait être. Il apparaît clairement qu'il ne peut être question de 'difficile à digérer', mais qu'il doit s'agir de 'qui aura un destin difficile'. Ce qui revient à dire que la leçon de $\Pi$ est juste, contre celle de $\Omega$.

Quant aux leçons qu'il est possible de retrouver à partir d'accidents du Parisinus, nous en donnons ici un exemple:

\section{Th. 54:}

Le contexte dans lequel intervient le terme étudié ici est celui des mesures à prendre pour repousser les serpents; parmi celles-ci, le jonchage avec des plantes odorantes. A priori, la leçon de $\Omega$ semble devoir être préférée, à tout le moins

87 C'est à Cazzaniga 1963, p. 85, que revient le mérite d'avoir déterminé que la leçon de $\Pi$ en ce passage est correcte.

88 C'est Jacques 1955 , p. 16, note $a d$ v. 152 qui a signalé l'intérêt de cette leçon du Parisinus.

89 Geymonat 1974, p. 119.

90 P. 70, 1. 15-20, éd. Papathomopoulos. 
parce qu'elle constitue un mot attesté et est confirmée par la scolie ${ }^{91}$; de plus, son sens correspond bien à ce que nécessite le passage; il pourrait donc s'agir d'odeur à fuir, s'il n'y avait que, dans ses autres attestations, le terme est utilisé pour définir les endroits vers où l'on fuit. $\mathrm{D}$ 'autre part, la leçon de $\Pi$ désigne une 'tablette', une 'liste', une 'section' et est manifestement erronée. Puisque la paraphrase d'Euteknios n'est pas assez précise en ce passage pour nous aider ${ }^{92}$, l'on croit être condamné à l'aporie. Cependant, comme 1'a établi Cazzaniga ${ }^{93}$, la leçon de $\Pi$ doit être reprise, parce qu'elle peut faire l'objet d'une correction qui a quelque chance de correspondre au texte original et qui permet à tout le moins d'expliquer la genèse des leçons $\Omega$ et $\Pi$. En effet, si l'on accepte la leçon de $\Pi$ et si l'on y suppose une erreur de majuscule fondée sur le passage de $\mathrm{N}$ à

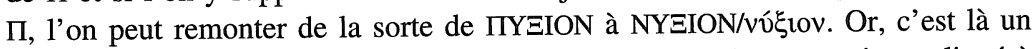
adjectif attesté par ailleurs, qui signifie 'piquant', littéralement; soit, appliqué à une odeur: 'forte'. Dans cette hypothèse, il nous faudrait expliquer la leçon de $\Omega$, qui pourrait être considérée comme une paradiorthose sur une leçon du type de celle de $\Pi$.

L'usage du texte du Parisinus qu'il conviendra d'avoir désormais sera donc différencié selon les cas, sans pouvoir plus être, cependant, aussi important qu'il le fut jusqu'ici. Ainsi réévalué, le Parisinus sera à considérer comme un témoin des vicissitudes subies par les poèmes de Nicandre dans les mondes tardo-antique et byzantin, avec, comme dans tous les autres textes, de bonnes leçons maintenues, mais aussi des gloses, commentaires, paradiorthoses et autres interventions toutes confondues, qui provoquèrent sans aucun doute cette impression de supériorité du manuscrit, alors qu'il s'agit plutôt d'une très forte différence.

\section{Nouvelles perspectives pour la lexicologie nicandréenne et grecque antique.}

Ce rééquilibrage de l'usage du Parisinus dans l'établissement du texte de Nicandre n'est pas sans conséquences sur la lexicologie nicandréenne et, de façon plus générale, sur celle de la langue grecque, d'époque autant hellénistique que tardo-antique et proto-byzantine, ainsi que sur l'histoire du lexique poétique grec et, notamment, de ses relations avec le lexique scientifique. Cet impact ira de la modification de la liste des occurrences d'unités lexicales jusqu'à la disparition de certaines, ou, à tout le moins, jusqu'à un changement de leur statut dans la langue grecque: non plus des leçons hellénistiques authentiques, mais des créations d'érudits tardo-antiques ou protobyzantins, à

91 Crugnola 1971, p. 53.

92 P. 6, 1. 27 - 7, 1. 2, éd. Papathomopoulos.

93 Cazzaniga 1960, p. 188-190. 
moins que ce ne soient des résultats d'accidents de la tradition manuscrite. Quoique les cas d'ecdotique analysés ci-dessus permettent déjà de percevoir le fait, nous voudrions le mettre en évidence de façon quelque peu plus spécifique par des cas significatifs.

Th. 131: ó $\delta \grave{\alpha} \xi \Omega, \dot{\alpha} \mu \grave{v} \xi \Pi$

Dans ce passage (vers 128-134), Nicandre décrit l'accouplement prétendûment sauvage des vipères, qui se solde par la décapitation du mâle par la femelle. Les deux adverbes ont la même signification ('en mordant'); le premier semble appartenir à la poésie homérique et à la tragédie, et renvoie à un verbe courant utilisé par Nicandre (ó $\delta \dot{\xi} \xi \omega, T h .306$ ), alors que le second semble rare (il ne serait attesté qu'en une unique autre occurrence) et est construit sur un verbe d'usage courant, qui fut toutefois utilisé en poésie, mais avec un sens métaphorique. Peut-être faut-il favoriser la leçon $\Omega$ et considérer celle de $\Pi$ comme une falsa lectio à éliminer du lexique nicandréen.

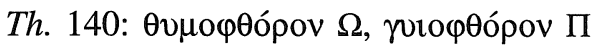

L'adjectif dont nous cherchons à déterminer ici quel il doit être, est appliqué au venin des serpents, de façon générale. La leçon de $\Omega$ ('qui détruit la vie', littéralement) reprend un adjectif homérique et hésiodique et rappelle un usage homérique, puisque, dans une attestation de l'Odyssée à tout le moins, il est

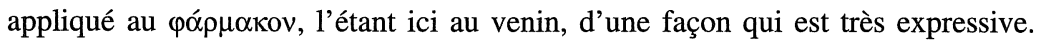
Quant à l'adjectif $\Pi$, il semble constituer une attestation unique et présente un sens concret ('qui détruit les membres') sans doute moins en accord avec les choix lexicaux de Nicandre. Il faut probablement le considérer comme une variante post-classique de la leçon précédente, de formation certes correcte, mais d'origine non identifiée jusqu'à plus ample information.

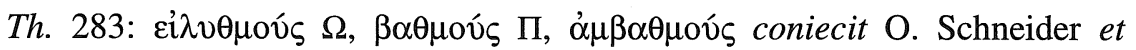
receperunt Gow et Scholfield

Nicandre décrit le serpent haimorroos (vers 282-285), qui, dit-il, dort dans des endroits pierreux (283: $\pi \varepsilon \tau \rho \omega ́ \delta \varepsilon \alpha \varsigma)$ qualifiés par l'adjectif que nous cherchons à identifier ici. La leçon $\alpha \mu \beta \alpha \theta \mu$ oús est une conjecture d'O. Schneider obtenue à partir de l'incompréhensible $\beta \alpha \theta \mu$ ov́ $\varsigma$ du Parisinus seul, par apocope et assimila-

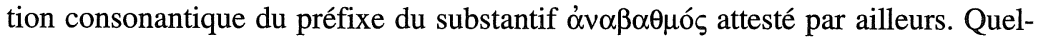
que vraisemblable qu'elle soit dans son traitement phonétique, cette leçon pose cependant un problème de sémantique, puisqu'elle désigne la 'marche d'un escalier', et semble ne pas pouvoir être préférée à la leçon de $\Omega$ qui fournit un sens

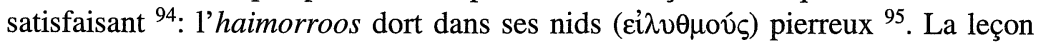
proposée par $\mathrm{O}$. Schneider est sans doute à considérer désormais comme une

94 Cette leçon est confirmée par la scolie (p. 130 éd. Crugnola, sub 283 b), sans l'être par Euteknios, par trop imprécis sur ce passage (p. 17, 1. 26-28 éd. Papathomopoulos).

95 Dans le même sens, voir White 1987, p. 19. 
conjecture d'érudit moderne, voire, plus précisément, comme une paradiorthose qui n'a pas d'argument textuel en sa faveur; à ce titre, elle appartient à l'histoire de la philologie et non à la lexicologie grecque.

\section{Th. 558: $\lambda \alpha ́ \zeta$ oเo $\Omega, \lambda \varepsilon ́ \psi$ oเo $\Pi$}

Dans la préparation d'un remède destiné à traiter les envenimements, Nicandre décrit cette opération qui consiste à prélever les membranes d'un cerveau de poule

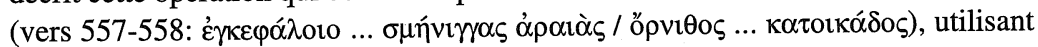
pour ce faire le verbe à l'impératif sur lequel les leçons de $\Omega$ et de $\Pi$ diffèrent.

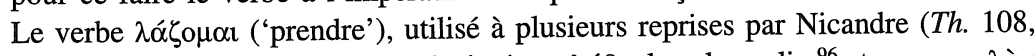

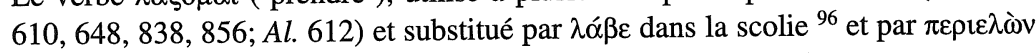
chez Euteknios ${ }^{97}$, est d'un usage courant dans la poésie homérique, se retrouvant aussi dans le Corpus Hippocraticum. Quant au verbe $\lambda \varepsilon ́ \pi \tau \omega$ de $\Pi$, il présente un sens qui ne convient peut-être pas bien au passage, puisqu'il signifie fondamentalement 'amincir'. Ceci ne modifiera pas le lexique grec, mais la liste des occurrences de deux de ses unités, constituant par ailleurs un parmi les nombreux cas de l'homérisme de Nicandre; à ce titre, ce passage, comme tous les autres reposant sur le même principe de sélection lexicale, illustrera l'histoire du lexique grec, avec notamment des reprises d'unités antiques par des auteurs-érudits, lexicologues et lexicographes, et collectionneurs d'antiquités.

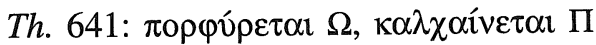

Dans la description des deux vipérines (vers 636-644), Nicandre précise ici la couleur d'une espèce. Le verbe de la leçon $\Omega$ est présent dans la poésie homérique où il signifie 'être troublé'; il semble avoir été réactualisé à l'époque hellénistique, mais avec un nouveau sens, qui convient bien ici: 'être rouge'. Quant au verbe $\Pi$, il apparaît dans le théâtre et chez Lycophron, avec le sens de 'être agité, s'inquiéter de'. Le sens qu'il devrait avoir ici ('être' ou 'devenir rouge') n'est attesté nulle part ailleurs et fait naître le soupçon que la leçon de $\Pi$ pourrait être née d'un sustantif $\chi \alpha \lambda$ кós ('le cuivre', avec sa couleur typique) éventuellement placé entre les lignes et puis assimilé dans le texte où il fut transformé en verbe, avec une modification en conséquence de façon à correspondre à une unité attestée du lexique grec. Quoi qu'il en soit de cette hypothèse sur sa génétique, ce terme n'est sans doute pas à recevoir dans le lexique grec hellénistique, ni tel quel ni pourvu du sens qu'il devrait avoir ici, mais est à considérer comme le résultat d'un accident de tradition, qui a conduit à une superposition indue avec un verbe attesté par ailleurs.

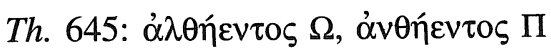

Dans une énumération d'ingrédients pour des remèdes contre les envenimements, Nicandre cite l'ókóvӨov, déterminé par l'adjectif dont il est question ici.

96 P. 213, 1.18 éd. Crugnola.

97 P. 32, 1. 20 éd. Papathomopoulos. 
Celui attesté par $\Omega$ apparaît déjà au vers 84 des Thêriaka et offre un sens qui convient bien au passage ('salutaire'), tandis que celui de $\Pi$ ('fleuri'), quoiqu'il soit imagé, est cependant banal ici; par ailleurs, la notion de 'fleuri' est attestée au vers 610 des Alexipharmaka avec l'adjectif óv $\theta \tilde{n} \mu \omega v$, formé sur la même racine, mais avec une composition morphologique plus recherchée. On adoptera donc la leçon de $\Omega$ et l'on changera en conséquence la liste des occurrences des deux adjectifs.

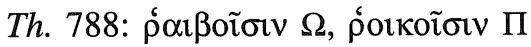

Lorsqu'il énumère et décrit les scorpions venimeux, Nicandre en mentionne un qui, dit-il, ressemble au crabe (vers 788-900). Dans ce contexte apparaît le mot discuté ici: les deux leçons ont un même sens ('recourbé', 'tordu'), mais semblent appartenir à des niveaux de langue différents: celle de $\Omega$ n'apparait, avant Nicandre, que chez Aristophane et Lycophron, tandis que celle de $\Pi$, attestée chez Archiloque et Théocrite, est utilisée aussi dans le Corpus hippocraticum et chez Aristote. Contre la version de $\Omega$, il faut peut-être relever que le terme est utilisé dix vers plus bas (799), en une leçon attestée autant par $\Omega$ que $\Pi$.

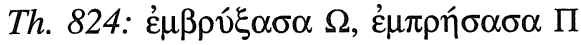

Nous avons vu que la leçon de $\Omega$ doit sans doute être préférée à celle de $\Pi$. Apparaissant plus haut dans les Thêriaka, vers 271, ainsi que dans les Alexipharmaka, vers 338, elle semble être une création de Nicandre, au contraire de la seconde qui fut d'un usage extrêmement courant, voire banal. On ajoutera donc désormais ce passage à la liste des occurrences de ce qui, dans l'état actuel de la documentation, semble être un néologisme de notre auteur.

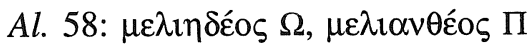

L'adjectif dont nous donnons ici les deux variantes apparaît dans l'énumération des remèdes destinés à traiter l'empoisonnement dû à ce que les Grecs appelaient l'akoniton, qui ne semble pas être notre aconit. Il détermine le terme olvns et appartient, pour la variante de $\Omega$ ('doux comme le miel'), à la poésie homérique, lyrique et pindarique, tandis que la variänte de $\Pi$ ('aux fleurs de miel', 'fleuri comme le miel') ne semble pas attestée ailleurs. Outre qu'elle ne donne pas un sens satisfaisant, cette dernière leçon n'est pas attestée par la scolie, au contraire

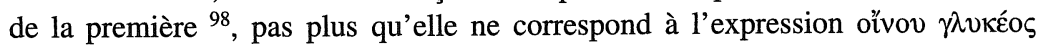
d'Euteknios ${ }^{99}$, qui est l'exact équivalent de la leçon de $\Omega$. Il convient donc de faire disparaître la leçon de $\Pi$ du lexique hellénistique et la faire passer dans le secteur de l'histoire des textes, où elle figurera parmi les accidents de transmission, sans doute au titre de correction savante.

98 P. 51, sub 58 c, éd. Geymonat.

99 P. 58, 1. 9, éd. Papathomopoulos. 


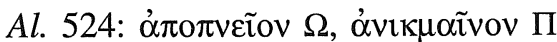

Dans le contexte de la description des champignons qui deviennent vénéneux lorsqu'ils croissent sur des tanières de serpents (vers 523-526), ces participes qualifient le venin: le premier le décrit qui 's'exhale' et le second qui 's'évapore', si tel est bien le sens du dernier, puisqu'il semble être un unicum, même s'il se rapproche de la forme $\dot{\alpha} v 1 \kappa \mu \alpha \dot{\alpha} \zeta \omega$; mais peut-être constitue-t-il une falsa lectio, et d'autant plus que la scolie donne la leçon de $\Omega{ }^{100}$, tandis qu'Euteknios paraphra-

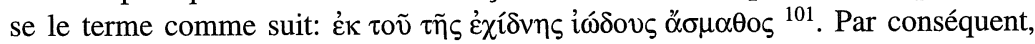
la leçon de $\Pi$ doit être écartée, n'étant certes pas un hapax legomenon créé par Nicandre, mais le résultat d'un accident de transmission textuelle; de ce fait, elle ne doit pas figurer dans le lexique de la langue grecque d'époque hellénistique, mais appartient à la langue érudite de l'Antiquité tardive ou du monde protobyzantin.

\section{Al. 552: $\psi \alpha \varphi \alpha \rho \circ \tilde{\sigma} \sigma \mathrm{\Omega}, \psi \alpha \theta v \rho \circ \tilde{\sigma} \sigma \mathrm{V} \Pi$}

De ces deux adjectifs descriptifs, le premier évoque la couleur pâle et le second l'état friable. Appliqués, comme c'est le cas ici, à la farine, le premier, attesté aussi par la scolie ${ }^{102}$, est plus indiqué que le second, dont on ne voit pas bien, a priori, la relation avec la farine. Il convient de changer en conséquence la liste des occurrences des deux termes.

\section{Al. 605: $\dot{\alpha} \lambda \hat{\imath} \beta \alpha \pi \tau$ ov $\Omega, \dot{\alpha} \lambda \hat{\imath} \beta \lambda \alpha \pi \tau$ ○ $\Pi$}

Le dernier cas que nous citerons est sans aucun doute le plus remarquable. Le passage (vers 604-606) se rapporte à l'ache des marais, que Nicandre prescrit pour traiter l'empoisonnement provoqué par la litharge (vers 594-610).

Pour pouvoir déterminer laquelle des deux leçons doit être adoptée, il convient d'en définir avec précision le contexte: l'ache des marais étant utilisée pour couronner les vainqueurs des Jeux Isthmiques, Nicandre dessine au départ de sa mention un petit tableau mythologique, qui évoque la fondation des jeux. C'est ainsi qu'il évoque l'histoire de celui en l'honneur de qui étaient célébrés ces jeux, Mélikertês, dont la légende veut qu'il fût précipité dans l'Egée par sa mère et mourut de la sorte.

C'est à son nom que se rapporte l'adjectif pour lequel nous avons les deux variantes données ci-dessus. Il est certainement possible de les expliquer par des mécanismes de confusions de majuscule, puisqu'ils sont isographes, à l'exception du lambda supplémentaire du second, et puisque celui-ci peut s'expliquer par un redoublement du A devenu ensuite $\Lambda$ (AA $>\Lambda \mathrm{A})$. Mais l'on pourrait aussi poser le contraire, avec l'apparition du premier adjectif par simplification du groupe $\Lambda \mathrm{A}$, qu'il ait ou non donné lieu à une géminée par suite de la confusion de ses

100 P. 181, , sub 584 a et b, éd. Geymonat.

101 P. 80, 1. 22-23, éd. Papathomopoulos.

102 P. 190, sub 552 c et d, éd. Geymonat. 
deux lettres, avec, par après, simplification $(\Lambda \mathrm{A}>\mathrm{AA}>\mathrm{A})$. Soit les trois possibilités suivantes:

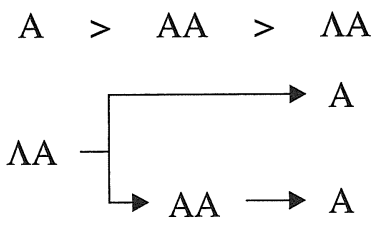

Cependant, outre que la seconde leçon ne semble attestée nulle part ailleurs, la première appartient à la poésie lyrique où elle désigne un oiseau marin. Appliquée ici à Mélikertês, elle crée un effet sémantique pas dénué de finesse: certes, l'adjectif $\dot{\alpha} \lambda \hat{i} \beta \alpha \pi \tau$ v signifiera 'plongé dans la mer', selon une interprétation attentive à identifier précisément la valeur exacte de chacun des composants; mais, simultanément, par la superposition avec le sens lyrique, il fait de Mélikertês comme un oiseau marin en une comparaison qui se fait métaphore. La leçon de $\Pi$, quant à elle, est privée de ces potentialités d'évocation, puisque, s'il faut la décomposer étymologiquement, elle signifie: 'blessé par la mer'; elle a donc quelque probabilité d'être une déformation de la première et doit être considérée dorénavant comme une falsa lectio produite par la tradition manuscrite. A ce titre, elle ne doit plus figurer ni dans le lexique de Nicandre ni dans celui de la langue grecque d'époque hellénistique, mais doit rentrer dans le monde des leçons érudites des éditions antiques, avec leurs commentaires.

\section{Conclusions.}

La bonne trentaine de cas présentés ici et prélevés parmi tant d'autres non moins démonstratifs, est de nature à mettre en lumière l'exacte nature du texte du Parisinus: s'écartant en plus d'une leçon de celui des autres manuscrits unanimes, il est moins un meilleur reflet du texte original que le résultat d'une révision. Les interventions explicatives qui le caractérisent furent sans doute portées dans un premier temps dans les marges de la page ou entre les lignes du texte et se seront substituées ensuite aux lemmes auxquels elles se rapportaient. De la sorte, le texte du Parisinus se transforma en un réel méta-texte fait de deux niveaux de lecture qui ont perdu leur articulation logique et se sont superposés au point de se confondre. Sans aucun doute plus compréhensible et moins énigmatique pour cette raison même, le texte du Parisinus a perdu en authenticité dans le même temps, semblant, par contre, de meilleure qualité aux éditeurs modernes.

Contrairement à ce que voulut $\mathrm{O}$. Schneider suivi par Gow et Scholfield, nombre de leçons du manuscrit ne devront plus être reçues dans le texte de Nicandre, pas plus que dans le lexique de notre auteur ou dans celui du grec d'époque hellénistique, mais sont appelées à figurer dans le lexique de l'époque de la constitution du texte du Parisinus, dont on ne sait, dans l'état actuel 
des travaux, quelle elle fut; cependant, elle dut être antérieure à la graphie minuscule et, donc, au IX ${ }^{\text {ème }}$ siècle, et pourrait être située plus vers la fin de l'Antiquité tardive ou les débuts de l'époque proto-byzantine, qui semble avoir favorisé et la poésie didactique et la paraphrase de nos oeuvres et d'autres semblables. Ceci pour autant que les leçons du Parisinus rejetées de l'édition du texte de Nicandre relèvent du champ lexical et non de celui de l'ecdotique.

Quoi qu'il en soit de ce point, le statut du Parisinus change dans l'histoire du texte de Nicandre, à tout le moins en partie: de source directe du texte qu'il était jusqu'ici, il devient un corpus de gloses qu'il conviendra désormais de faire figurer comme telles dans le lexique de notre auteur et de la langue grecque d'époque tardive, en regard des termes plus authentiquement nicandréens auxquelles elles se rapportent.

AlaIN TOUWAIDE

\section{Appendice bibliographique}

Nous reprenons ici les travaux essentiels et récents indispensables pour l'étude du texte de Nicandre et de son histoire. Pour la clarté, nous les avons répartis par catégories que nous avons identifiées par des titres en gras; et, à l'intérieur de celles-ci, nous avons signalé les travaux selon leur ordre chronologique de publication.

Cet inventaire n'est pas exhaustif, surtout pour les Travaux et études, mais reprend les publications mentionnées dans l'article et vise à constituer un outil de travail temporaire, en attendant la publication de la bibliographie chronologique et analytique des travaux consacrés aux poèmes de Nicandre que nous avons mise en chantier. Sur ce point, il convient de signaler dès à présent qu'elle ne portera pas seulement sur les publications relatives aux questions de tradition manuscrit, d'ecdotique ou de philologie, comme c'est le cas dans le présent article, mais inclura celles traitant des aspects du texte relevant de la fortuna de la littérature grecque antique, de l'histoire des sciences (zoologie, botanique, médecine, pharmacologie et thérapeutique), de l'illustration ou d'autres.

\section{Edition usuelle:}

Gow \& Scholfield 1953 - Nicander, The Poems and Poetical Fragments. Edited with a Translation and Notes by A. S. F. Gow and A. F. Scholfield, Cambridge, 1953.

\section{Editio princeps:}

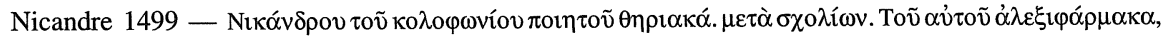
Venetiis, Apud Aldum, 1499.

\section{Editions modernes:}

J. S. Schneider 1792 - Nicandri, Alexipharmaca ..., ex libris scriptis emendavit ... J. G. Schneider, Halle, 1792.

J. G. Schneider 1816 - Nicandri Colophonii, Theriaca ..., ad librorum scriptorum fidem recensuit ... J. G. Schneider, Leipzig, 1816.

O. Schneider 1856 - Nicandrea, Theriaca et Alexipharmaca, recensuit et emendavit, ... O. Schneider, Leipzig, 1856. 


\section{Editions des paraphrases d'Euteknios}

Gualandri 1968 - Gualandri, I.: Eutecnii Paraphrasis in Nicandri Theriaca, (= Testi e documenti per lo studio dell'Antichità, 25), Milano et Varese, 1968.

Geymonat 1976 - Geymonat, M.: Eutecnii Paraphrasis in Nicandri Alexipharmaca, (= Testi e documenti per lo studio dell'Antichità, 57), Milano, 1976.

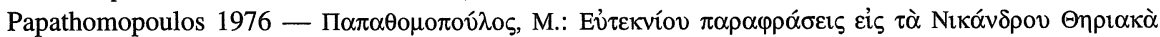

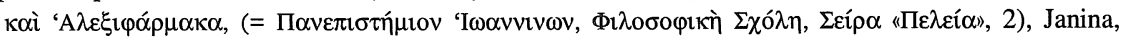
1976.

\section{Editions des scolies:}

Crugnola 1971 - Crugnola, A.: Scholia in Nicandri Theriaka cum glossis, (= Testi e documenti per lo studio dell'Antichità, 34), Milano e Varese, 1971.

Geymonat 1974 - Geymonat, M.: Scholia in Nicandri Alexipharmaca cum glossis, (= Testi e documenti per lo studio dell'Antichità, 48), Milano, 1974.

\section{Index et concordances:}

O. Schneider 1856 - Schneider, Otto, in Nicandrea (voir ci-dessus, sub 3), p. 312-346.

Berkowitz 1980 - Berkowitz, L.: A Concordance to Nicander (= TLG Publications Series, No 10), Irvine, 1980.

Papathomopoulos 1996 - Papathomopoulos, M.: Nicandri Theriacorum et Alexipharmacorum Concordantia, (Alpha-Omega, Reihe A: Lexica, Indizes, Konkordanzen zur klassischen Philologie, 91), Hildesheim, Zurich et New York, 1996.

\section{Etudes et travaux:}

Arrigoni 1970 - Arrigoni, G.: «Le Meleagridi in Antonio Liberale e Nicandro», Acme 23, 1970, pp. 17-28.

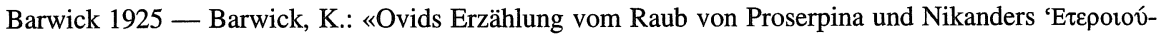
$\mu \varepsilon v \alpha »$, Hermes, 53 (= N. F. 34), 1925, pp. 110-112.

Bodson 1986 - Bodson, L.: «Observations sur le vocabulaire de la zoologie antique: les noms de serpents en grec et en latin», GRECO -Documents pour l'histoire du vocabulaire scientifique 8, 1986, pp. 65-119.

Brenning 1904 - Brenning, M.: «Nikanders Theriaka und Alexipharmaka», Allgemeine Medicinische Central-Zeitung 73, 1904, pp. 112-114; 132-134; 327-330; 346-349; 368-371; 387-390.

Bussemaker 1849 - Scholia et paraphrases in Nicandrum, éd. U. C. Bussemaker, dans Scholia in Theocritum, Nicandrum et Oppianum, Paris, 1849, pp. 202-219 et 411-418.

Cazzaniga 1956 - Cazzaniga, I.: «I Theriaka Nicandri dell'Ambrosiano C 32 Sup. (sec. XV)», Acme 9, 1956, pp. 49-51.

Cazzaniga 1956/2 - Cazzaniga, I.: Glosse inedite ai Theriaka ed Alexipharmaka di Nicandro (Cod. Ambrosiano C 32 Sup.), Pise, 1956.

Cazzaniga 1956/3 - Cazzaniga, I.: «Nuovo frammento di scolion a Nicandro, Theriaka, vv. 526-529», Studi italiani di filologia classica 27-28, 1956, pp. 83-101.

Cazzaniga 1957 - Cazzaniga, I.: «Note critiche e filologiche: Nicandro, Theriaka 406», La parola del passato 12, 1957, pp. 130-131.

Cazzaniga 1959 - Cazzaniga, I.: «Alcuni ‘colori’ nicandrei in Stazio e Claudio (Theb. V, 505; Gigant., II, 25)», Acme 12, 1953, pp. 125-129.

Cazzaniga 1960 - Cazzaniga, I.: «A proposito di una presunta ironia vergiliana», Studi italiani di filologia classica N.S. 32, 1960, pp. 1-14.

Cazzaniga 1960/2 - Cazzaniga, I.: «Osservazioni critico-testuali ad alcuni passi Nicandrei (Ther. 308, Alex. 64, 150, 278, 332, 565, 575)», dans Etudes en l'honneur de L. Castiglioni, Florence, 1960, vol. 1, pp. 179-198.

Cazzaniga 1960/3 - Cazzaniga, I.: «Colori Nicandrei in Virgilio», Studi italiani di filologia classica N.S. 32 , 1960, pp. 18-37. 
Cazzaniga 1963 - Cazzaniga, I.: «Osservazioni al testo ed alla tradizione manoscritta dei Theriaka Nicandrei: I», Studi classici e orientali 12, 1963, pp. 84-92.

Cazzaniga 1964 - Cazzaniga, I.: «Note critico-testuali a due passi degli scholia ai Theriaka Nicandrei, I», Maia 16, 1964, pp. 389-394.

Cazzaniga 1966 - Cazzaniga, I.: «Nota critico-testuale a scholion Nicandri Theriaca 102 e 662», Rendiconti dell'Istituto Lombardo, Accademia di scienze e lettere, Classe di lettere e scienze morali e storiche 100, 1966, pp. 276-278.

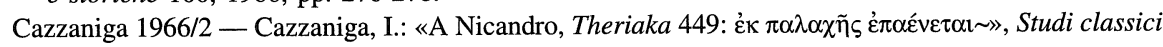
e orientali 15,1966, pp. 281-283.

Cazzaniga 1972 - Cazzaniga, I.: «L'inno di Nicandro ad Attalo I (frg. 104). Esegesi e problematica», La parola del passato 27, 1972, pp. 369-396.

Cazzaniga 1973 - Cazzaniga, I.: «Observationes criticae in Nicandri Theriaka», Studi orientali e classici 22,1973 , pp. 50-84.

Crugnola 1962 - Crugnola, A.: «La lingua poetica di Nicandro», Acme 14, 1962, pp. 119-152.

Geymonat 1970 - Geymonat, M.: «Spigolature Nicandree», Acme 23, 1970, pp. 137-143.

Gow 1951 - S. F. Gow, A.: «Nicandrea», Classical Quarterly 45, 1951, pp. 95-118.

Gualandri 1970 - Gualandri, I.: «Nota esegetica ad Eneide 2, 471-472», Acme 23, 1970, pp. 149-151.

Jacques 1955 - Jacques, J.-M.: «Les Alexipharmaques de Nicandre», Revue des études anciennes 57, 1955, pp. 5-35.

Jacques 1969 - Jacques, J.-M.: «Aratos et Nicandre», Revue des études anciennes 71, 1969, pp. 38-56.

Jacques 1979 - Jacques, J.-M.: «Nicandre de Colophon poète et médecin», KTEMA 4, 1979, pp. 133-149.

Jacques 1984 - Jacques, J.-M.: «Nicandre (Alexipharmaques, 611-615), Callimaque (Fr. 659 Pfeiffer) et le témoignage d'Andréas sur l'if de l'Oeta», dans Studi in onore di Adelmo Barigazzi, Rome, 1984, vol. 1, pp. 299-307.

Knoefel \& Covi 1991 - Knoefel, P. K. - Covi, M. C.: A Hellenistic Treatise on Poisonous Animals (The Theriaca of Nicander of Colophon). A Contribution to the History of Toxicology, Lewiston, Queenston et Lampeter, 1991.

Scarborough 1977 - Scarborough, J.: «Nicander's Toxicology, I: Snakes», Pharmacy in History 19, 1977, pp. 3-23.

Scarborough 1979 - Scarborough, J.: «Nicander's Toxicology, II: Spiders, Scorpions, Insects and Myriapods», Pharmacy in History 21, 1979, pp. 3-34; 73-92.

Skaltsa-Diamantis \& Philianos 1994 - Skaltsa-Diamantis, H. -Philianos, S.: «Aspects pharmacognostiques des Thériaka de Nicandre de Colophon», Atti e Memorie della Accademia italiana di Storia della Farmacia 11, 1994, pp. 139-143.

Touwaide 1975 - Touwaide, A.: Les Theriaca de Nicandre, Louvain, 1975 (thèse dactylographiée).

Touwaide 1977 - Touwaide, A.: Les Alexipharmaca de Nicandre, 2 vol. (s. 1. n. d. [en fait: Bruxelles, 1977]) (thèse dactylographiée).

Touwaide 1991 - Touwaide, A.: «Nicandre: de la science à la poésie - Contribution à l'exégèse de la poésie médicale grecque», Aevum 65, 1991, pp. 65-101.

Touwaide 1991/2 - Touwaide, A.: «La toxicologie des poisons dans l'Antiquité et à Byzance Introduction à une étude systémique», Revue d'histoire de la pharmacie 290, 1991, pp. 265-281.

Touwaide 1991/3 - Touwaide, A.: «Panorama des recherches en histoire de la médecine intéressant la toxicologie depuis 1970», Lettre d'information du Centre Jean-Palerne 19, 1991, pp. 8-26.

Touwaide 1997 - Nicandre, Thêriaka et Alexipharmaka. Reproduction en fac-similé du manuscrit Parisinus Bibliothecae Nationalis Supplementum graecum 247, Barcelona, 1997.

Touwaide 1997/2 - Nicandro, Thêriaka et Alexipharmaka. Reproducción facsímil del manuscrito Parisinus Bibliothecae Nationalis Supplementum graecum 247, Barcelona, 1997.

Vera 1970 - Vera, F.: Científicos griegos. Recopilación, estudio preliminar, preámbulos y notas por -, vol. 2, Madrid, 1970.

White 1987 - White, H.: Studies in the Poetry of Nicander, (= Classical and Byzantine Monographs, 12), Amsterdam, 1987. 